\title{
Determining pressure-temperature phase diagrams of materials
}

Article

Accepted Version

Baldock, R. J. N., Partay, L. B., Bartok, A. P., Payne, M. C. and Csanyi, G. (2016) Determining pressure-temperature phase diagrams of materials. Physical Review B, 93. 174108. ISSN 1098-0121 doi: https://doi.org/10.1103/PhysRevB.93.174108 Available at https://centaur.reading.ac.uk/67188/

It is advisable to refer to the publisher's version if you intend to cite from the work. See Guidance on citing.

To link to this article DOI: http://dx.doi.org/10.1103/PhysRevB.93.174108

Publisher: American Physical Society

All outputs in CentAUR are protected by Intellectual Property Rights law, including copyright law. Copyright and IPR is retained by the creators or other copyright holders. Terms and conditions for use of this material are defined in the End User Agreement.

\section{www.reading.ac.uk/centaur}

\section{CentAUR}

Central Archive at the University of Reading

Reading's research outputs online 


\title{
Determining pressure-temperature phase diagrams of materials
}

\author{
Robert J. N. Baldock ${ }^{1},{ }^{*}$ Lívia B. Pártay ${ }^{2}$, Albert P. Bartók ${ }^{3}$, Michael C. Payne ${ }^{1}$, and Gábor Csányi ${ }^{3}$ \\ ${ }^{1}$ Cavendish, ${ }^{2}$ University Chemical and ${ }^{3}$ Engineering Laboratories, \\ University of Cambridge, Cambridge, United Kingdom
}

(Dated: September 29, 2016)

\begin{abstract}
We extend the nested sampling algorithm to simulate materials under periodic boundary and constant pressure conditions, and show how it can be used to determine the complete equilibrium phase diagram, for a given potential energy function, efficiently and in a highly automated fashion. The only inputs required are the composition and the desired pressure and temperature ranges, in particular, solid-solid phase transitions are recovered without any a priori knowledge about the structure of solid phases. We benchmark and showcase the algorithm on the periodic Lennard-Jones system, aluminium and NiTi.
\end{abstract}

\section{INTRODUCTION}

Phase diagrams of materials describe the regions of stability and equilibria of structurally distinct phases and are fundamental in both materials science and industry. In order to augment experiments, computer simulations and theoretical calculations are often used to provide reference data and describe phase transitions. A plethora of methods exist to determine individual phase boundaries, including Gibbs ensemble Monte Carlo [1], Gibbs-Duhem integration [2], thermodynamic integration and even direct molecular dynamics simulations of coexistence. Each of these algorithms requires the user to specify at least the identity and approximate location of the phase transition under investigation. Moreover, in the case of the solid phases, where much of the interest lies, advance knowledge of the crystal structure of each phase is required. Calculating an entire phase diagram by combining the results of such methods therefore demands a high degree of prior knowledge of the result. This in turn poses a barrier to the discovery of unexpected phases and phase transitions. Furthermore, such algorithms require specific expertise and separate setup for each type of phase transition.

In this paper we introduce a single algorithm, based on nested sampling (NS) [3, 4], that enables the efficient calculation of complete pressure-temperature phase diagrams, including the solid region. This algorithm requires no prior knowledge of the phase diagram, and takes only the potential energy function together with the desired pressure and temperature ranges as inputs. Moreover, the direct output of the simulation is the partition function as an explicit function of its natural variables, so calculating thermodynamic observables, such as the heat capacity, is straightforward.

Nested sampling systematically explores the entire potential energy landscape, and in this way is related to parallel tempering (also known as replica exchange) $[5,6]$ and Wang-Landau sampling [7]. However, those algorithms encounter a particular convergence problem at

\footnotetext{
*rjnbaldock@gmail.com
}

first order phase transitions because the probability distributions (parametrised in terms of temperature in case of parallel tempering or energy in case of Wang-Landau) on the two sides of the phase transition have very little overlap [8]. This results in poor equilibration between the distributions on either side of the phase transition and large errors (both random and systematic) in the predicted locations of phase transitions.

The NS algorithm was designed to solve this problem. It constructs a sequence of decreasing potential energy levels, $\left\{E_{i}\right\}$, each of which bounds from above a volume of configuration space $\chi_{i}$, with the property that $\chi_{i}$ is approximately a constant factor smaller than the volume, $\chi_{i-1}$, corresponding to the level above. Each volume is sampled uniformly, and therefore each distribution will have an approximately constant fractional overlap with the one immediately before and after, ensuring fast convergence of the sampling and allowing an accurate evaluation of phase space integrals. In particular, the energy levels near the phase transition, where phase volumes change rapidly, will be very narrowly spaced. The sequence of energy levels comprise a discretisation of the cumulative density of states $\chi(E)$, which allows the evaluation of the partition function at arbitrary temperatures,

$$
\begin{aligned}
Z(N, V, \beta) & =\frac{1}{N !}\left(\frac{2 \pi m}{\beta h^{2}}\right)^{3 N / 2} \int d E \chi^{\prime}(E) e^{-\beta E} \\
& \approx Z_{m}(N, \beta) \sum_{i}\left(\chi_{i-1}-\chi_{i}\right) e^{-\beta E_{i}}
\end{aligned}
$$

where $N$ is the number of particles of mass $m, V$ is the volume, $\beta$ is the inverse temperature, $h$ is Planck's constant, the density of states $\chi^{\prime}$ is the derivative of $\chi$, and we labelled the factor resulting from the momentum integral as $Z_{m}$. The total phase space volume is $\chi_{0}=V^{N}$ corresponding to the ideal gas limit. Note that the sequence of energies $\left\{E_{i}\right\}$ and configuration space volumes $\left\{\chi_{i}\right\}$ are independent of temperature, so the partition function can be evaluated a posteriori at any temperature by changing $\beta$ in (2).

The basic NS algorithm is as follows. We initialise by generating a pool of $K$ uniformly random configurations and iterate the following loop starting at $i=1$. 
1. Record the energy of the sample with the highest energy as $E_{i}$, and use it as the new energy limit, $E_{\text {limit }} \leftarrow E_{i}$. The corresponding phase space volume is $\chi_{i} \approx \chi_{0}[K /(K+1)]^{i}$.

2. Remove the sample with energy $E_{i}$ from the pool and generate a new configuration uniformly random in the configuration space, subject to the constraint that its energy is less than $E_{\text {limit }}$. One way to do this is to clone a randomly chosen existing configuration and make it undergo a random walk of $L$ steps, subject only to the energy limit constraint.

\section{Let $i \leftarrow i+1$, and return to step 1 .}

At each iteration, the pool of $K$ samples are uniformly distributed in configuration space with energy $E<E_{\text {limit }}$. The finite sample size leads to a statistical error in $\log \chi_{i}$, and also in the computed observables, that is asymptotically proportional to $1 / \sqrt{K}$, so any desired accuracy can be achieved by increasing $K$. Note that for any given $K$, the sequence of energies and phase volumes converge exponentially fast (the number of iterations required to obtain results shown below never exceeded $2000 \cdot K$ ), and increasing $K$ necessitates a new simulation from scratch.

Since its inception NS has been used successfully for Bayesian model selection in astrophysics [9], and also to investigate the potential energy landscapes of atomistic systems ranging from clusters to proteins [10? -17].

The structure of this paper is as follows. In section II we modify the NS algorithm to enable its application at constant isotropic pressure with fully flexible periodic boundary conditions [18] where the periodic simulation cell is allowed to change shape. In sections III and IV we show that this development enables the determination of pressure-temperature phase diagrams of materials directly from the potential energy function without recourse to any other a priori knowledge. In particular, in section IV we calculate phase diagrams for aluminium and NiTi. Finally in section V we conclude this paper, discussing some consequences of the capability to calculate entire phase diagrams with a single method and in a highly automated fashion.

\section{NESTED SAMPLING WITH FULLY FLEXIBLE PERIODIC BOUNDARY CONDITIONS AT CONSTANT PRESSURE}

Nested sampling produces new samples by cloning an existing sample and then evolving the clone using a Markov chain Monte Carlo (MCMC) random walk [19]. Although one could work in the NVT ensemble and use equations 1 and 2, that would be very inefficient. MCMC simulations performed at fixed pressure require just a fraction of the computational expense as equivalent calculations performed at fixed volume. There are two reasons for this.
First, allowing the system to change volume by dilating or contracting expedites the cooperative freeing of jammed atoms. In contrast, at fixed volume, atoms that have become jammed are only freed by the coincidental movement of all atoms to separate them. Consequently MCMC simulations at fixed pressure explore configuration space far more rapidly than simulations at fixed volume.

The second reason arises from the thermodynamic behaviour of systems at a first order phase transition. At a phase transition under constant volume conditions the two phases coexist and an interface forms between them. Such interfaces are large on the atomic scale [20] and the behaviour of atoms at an interface is not representative of the behaviour of atoms in the equilibrium phases. As a result the interface introduces a systematic error that is only overcome by simulating very large numbers of atoms.

Such interfaces also occur under constant pressure conditions in the infinite system size limit. The contribution to the Gibbs Free Energy from an interface is proportional to $\gamma N^{\frac{2}{3}}$, where $\gamma$ is the interfacial tension. In contrast, the Gibbs Free Energies of each of the pure phases are extensive (proportional to $N$ ). Therefore the Gibbs Free Energy cost of the interface is negligible for thermodynamic systems. Conversely, for the relatively small system sizes amenable to density of states calculation methods such as nested sampling, the Gibbs Free Energy cost of the interface is appreciable, provided $\gamma$ is not close to zero. Consequently, at a constant pressure phase transition between phases with identical atomic compositions, configurations containing an interface have negligible statistical weight in such simulations, and a discontinuous transition is observed from one equilibrium phase to the other. This enables the accurate simulation of phase transitions using much smaller numbers of atoms.

Using small numbers of atoms to simulate a phase transition naturally introduces new finite size errors. In particular, for a fixed number of atoms, it is not possible to represent all crystal structures in a simulation cell of fixed shape. This representational bias is removed by the use of fully flexible periodic boundary conditions [18], which allow the simulation cell to deform smoothly and thus take any shape. However, using fully flexible periodic boundary conditions allows the formation of very thin simulation cells containing unphysical quasi one and two dimensional configurations, characterised by interacting periodic images. In subsection II A we describe a rigorous solution to this new finite size problem. Later, in subsection IIB we describe the calculation of the constant pressure partition function and heat capacity, both as explicit functions of temperature, using nested sampling. 


\section{A. Constraint on the simulation cell to exclude unphysical quasi one and two dimensional configurations}

The partition function at fixed isotropic pressure $p$ with fully flexible periodic boundary conditions [18] is

$$
\begin{aligned}
\Delta(N, p, \beta)= & Z_{m} \beta p \int d \mathbf{h}_{0} \delta\left(\operatorname{det} \mathbf{h}_{0}-1\right) \times \\
& \int_{0}^{\infty} d V V^{N} \int_{(0,1)^{3 N}} d \mathbf{s} e^{-\beta H\left(\mathbf{s}, \mathbf{h}_{0}, V, p\right)} .
\end{aligned}
$$

Here $H\left(\mathbf{s}, \mathbf{h}_{0}, V, p\right)=E\left(\mathbf{s}, \mathbf{h}_{0}, V\right)+p V, \mathbf{h}$ is the $3 \times 3$ matrix of lattice vectors relating the Cartesian positions of the atoms $\mathbf{r}$ to the fractional coordinates $\mathbf{s}$ via $\mathbf{r}=\mathbf{h} \mathbf{s}$, $V=\operatorname{det} \mathbf{h}$ is the volume, and $\mathbf{h}_{\mathbf{0}}=\mathbf{h} V^{-1 / 3}$ is the image of the unit cell normalised to unit volume.

The partition function (3) corresponds to integration over all nine elements of the matrix $\mathbf{h}_{0}$, and the $\delta$-function restricts the integration to matrices satisfying $\operatorname{det} \mathbf{h}_{0}=1$. This partition function is formally correct in the thermodynamic limit $[18,21]$. However, finite systems in this description can adopt configurations for which the simulation cell becomes very thin. In this case, periodic boundary conditions give rise to a quasi one or two dimensional system. The prevalence of such configurations leads to a poor approximation of the three dimensional atomic system due to excessively large finite size effects. We exclude such thin configurations by changing the limits for integration over elements of $\mathbf{h}_{0}$, so that the perpendicular distances between opposite faces of the simulation cell $\mathbf{h}_{0}$ are greater than some "minimum cell depth" value $d_{0}$.

The perpendicular distance between faces of the unit cell $\mathbf{h}$ made by lattice vectors $\mathbf{h}^{(i)}$ and $\mathbf{h}^{(j)}$ is given by

$$
d_{\mathbf{h}^{(k)}}^{\perp}=\frac{\operatorname{det} \mathbf{h}}{\left|\mathbf{h}^{(i)} \times \mathbf{h}^{(j)}\right|} .
$$

The cell depth $D\left(\mathbf{h}_{0}\right)$, which measures how "thin" the cell has become, is defined as the minimum value of $d_{\mathbf{h}^{(k)}}^{\perp}$, for the cell at normalised (unit) volume $\mathbf{h}_{0}$.

$$
D\left(\mathbf{h}_{0}\right)=\min _{i=1,2,3}\left(d_{\mathbf{h}_{0}^{(i)}}^{\perp}\right)
$$

Thus we integrate over elements of $\mathbf{h}_{0}$ such that

$$
D\left(\mathbf{h}_{0}\right)>d_{0} .
$$

The minimum cell depth $d_{0}$ is a real number on the interval $[0,1]$ where $d_{0}=1$ restricts the simulation cell to a cube. Smaller values of $d_{0}$ are accordingly less restrictive on the shape of the simulation cell, and $d_{0}=0$ corresponds to no restrictions on the simulation cell.

Incorporating this change of integration limits into the partition function (3) yields a new partition function

$$
\begin{array}{r}
\widetilde{\Delta}\left(N, p, \beta, d_{0}\right)=Z_{m} \beta p \int_{D\left(\mathbf{h}_{0}\right)>d_{0}} d \mathbf{h}_{0} \delta\left(\operatorname{det} \mathbf{h}_{0}-1\right) \times \\
\int_{0}^{\infty} d V V^{N} \int_{(0,1)^{3 N}} d \mathbf{s} e^{-\beta H\left(\mathbf{s}, \mathbf{h}_{0}, V, p\right)} .
\end{array}
$$

In the thermodynamic limit (7) is equal to (3) up to a factor which depends only on $d_{0}$. The two partition functions are equal if and only if $d_{0}=0$.

In tests with 64 atoms we verified that the heat capacity curves were independent of $d_{0}$ at values of $0.65,0.7$ and 0.8 , in Lennard-Jonesium and aluminium. The window of independence from $d_{0}$ grows wider as the number of particles is increased. For larger numbers of atoms, there are more ways to arrange those atoms into a given crystal structure, including in simulation cells that are closer to a cube. Similarly, unphysical correlations are introduced when the absolute number of atoms between faces of the cell becomes too small, and therefore larger simulations can tolerate "thinner" simulation cells $\mathbf{h}_{0}$. The nickel-titanium calculations were performed with $d_{0}=0.7$.

\section{B. Partition function and thermodynamic variables}

The partition function we seek to calculate is given in equation (7). Above some sufficiently large volume $V_{0}$, we approximate the system as an ideal gas, neglecting interatomic interactions, which corresponds to the condition $E\left(\mathbf{s}, \mathbf{h}_{0}, V\right) \ll p V$. In this approximation the volume integral in (7) is the sum of two parts

$$
\begin{aligned}
& \widetilde{\Delta}\left(N, p, \beta, d_{0}\right) \approx Z_{m} \beta p\left[\Delta_{\mathrm{NS}}\left(N, p, \beta, V_{0}, d_{0}\right)\right. \\
& \left.+\int_{D\left(\mathbf{h}_{0}\right)>d_{0}} d \mathbf{h}_{0} \delta\left(\operatorname{det} \mathbf{h}_{0}-1\right) \int_{V_{0}}^{\infty} d V V^{N} \int_{(0,1)^{3 N}} d \mathbf{s} e^{-\beta p V}\right]
\end{aligned}
$$

where

$$
\begin{aligned}
\Delta_{\mathrm{NS}}\left(N, p, \beta, V_{0}, d_{0}\right) & =\int_{D\left(\mathbf{h}_{0}\right)>d_{0}} d \mathbf{h}_{0} \delta\left(\operatorname{det} \mathbf{h}_{0}-1\right) \times \\
& \int_{0}^{V_{0}} d V V^{N} \int_{(0,1)^{3 N}} d \mathbf{s} e^{-\beta\left[E\left(\mathbf{s}, \mathbf{h}_{0}, V\right)+p V\right]}
\end{aligned}
$$

We calculate $\Delta_{\text {NS }}$ using nested sampling. Calculations are performed at fixed pressure to generate a sequence of enthalpies, $H_{i}$, where $H=E\left(\mathbf{s}, V, \mathbf{h}_{0}\right)+p V$. The NS approximation for $\Delta_{\mathrm{NS}}$, is

$$
\begin{aligned}
\Delta_{\mathrm{NS}}\left(N, p, \beta, V_{0}, d_{0}\right) & \approx \sum_{i=1}^{i_{\max }}\left(\chi_{i-1}-\chi_{i}\right) e^{-\beta H_{i}} \\
& \approx \sum_{i=1}^{i_{\max }} \Delta \chi_{i} e^{-\beta H_{i}}
\end{aligned}
$$

where $\chi_{i} \approx \chi_{0}\left(\frac{K}{K+1}\right)^{i}, \chi_{0}=\frac{V_{0}^{N+1}}{N+1}$, and $\Delta \chi_{i} \approx \chi_{i-1}-$ $\chi_{i}$. We use single atom Monte Carlo (MC) moves in fractional coordinates with the amplitude updated every $\frac{K}{2}$ iterations to maintain a good acceptance rate. Uniform sampling of lattice shape matrices $\mathbf{h}_{0}$ subject to 
equation (6) was achieved by independent shearing and stretching moves which do not change the volume. The ratios of atom, volume, shear and stretch moves were $N: 10: 1: 1$. Further details of the MC moves and parallelisation scheme are given in the Supplemental Material (SM) [? ].

We show in appendix A that volumes greater than $V_{0}$ make a negligible contribution to the partition function (8), provided $k_{\mathrm{B}} T \ll p V_{0}$. In this case we have

$$
\widetilde{\Delta}\left(N, p, \beta, d_{0}\right) \approx \frac{\beta p}{N !}\left(\frac{2 \pi m}{\beta h^{2}}\right)^{3 N / 2} \Delta_{\mathrm{NS}}\left(N, p, \beta, V_{0}, d_{0}\right)
$$

where we have expanded $Z_{m}$. One can always assert the condition $k_{\mathrm{B}} T \ll p V_{0}$, and in practice it is easy to find values of $V_{0}$ suitable for physically relevant conditions. We found $V_{0}=10^{7} N \AA^{3}$ to be suitable for all conditions considered in this paper. From (11) we obtain the expected enthalpy

$$
\begin{aligned}
\langle H\rangle & =-\frac{\partial \log \widetilde{\Delta}\left(N, p, \beta, d_{0}\right)}{\partial \beta} \\
& =\left(\frac{3 N}{2}-1\right) \frac{1}{\beta}+\left\langle H_{\text {configurations }}\right\rangle
\end{aligned}
$$

and the heat capacity at constant pressure

$$
\begin{aligned}
C_{p}= & -k_{\mathrm{B}} \beta^{2} \frac{\partial\langle H\rangle}{\partial \beta} \\
= & \left(\frac{3 N}{2}-1\right) k_{\mathrm{B}} \\
& +k_{\mathrm{B}} \beta^{2}\left(\left\langle H_{\text {configurations }}^{2}\right\rangle-\left\langle H_{\text {configurations }}\right\rangle^{2}\right)
\end{aligned}
$$

where

$$
\begin{aligned}
\left\langle H_{\text {configurations }}\right\rangle & \approx \frac{\sum_{i=1}^{i_{\max }} \Delta \chi_{i} H_{i} e^{-\beta H_{i}}}{\sum_{i=1}^{i_{\max }} \Delta \chi_{i} e^{-\beta H_{i}}}, \\
\left\langle H_{\text {configurations }}^{2}\right\rangle & \approx \frac{\sum_{i=1}^{i_{\max }} \Delta \chi_{i} H_{i}^{2} e^{-\beta H_{i}}}{\sum_{i=1}^{i_{\max }} \Delta \chi_{i} e^{-\beta H_{i}}} .
\end{aligned}
$$

This form (15) naturally does not depend on the contribution made by the low density configurations omitted from the NS calculation, or explicitly on the value of $d_{0}$. We used equations (15) when calculating the heat capacities presented in this paper.

\section{CALCULATING PHASE DIAGRAMS}

In this section we describe a method for calculating the phase diagram of a material from the output of nested sampling. We then benchmark the performance of nested sampling on the periodic Lennard-Jones system, and find nested sampling to be orders of magnitude more efficient than Parallel Tempering (PT) for resolving the melting and evaporation transitions.

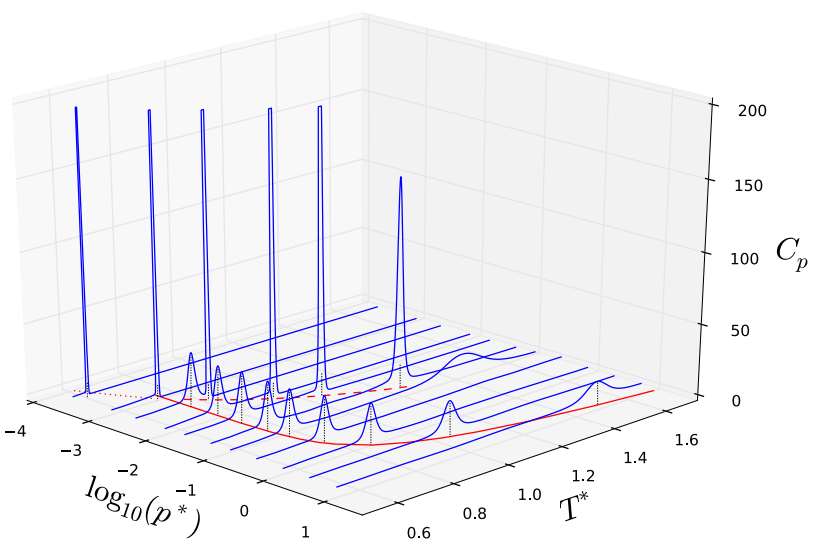

FIG. 1. Demonstration of how NS can be used to calculate phase diagrams, using the case of the periodic Lennard-Jones model. NS calculations are performed at a series of pressures and phase transitions are located by peaks of the heat capacity curves (blue). The red lines show values from the literature for the melting (solid) [23], boiling (dashed) [24] and sublimation (dotted) [25] curves.

Given the partition function (11), phase transitions can be easily located by finding the peaks of response functions such as the heat capacity (14). By performing separate NS simulations at a number of pressures and combining the pressure and temperature values corresponding to the heat capacity peaks one can straightforwardly construct the entire phase diagram including all thermodynamically stable phases. This process is illustrated in Figure ??.

In Figure ?? we compare the performance of NS to that of PT for calculating the melting and evaporation transitions. NS provides a reasonable estimate of the melting and boiling points using only $\sim 10^{8}$ energy evaluations, while parallel tempering needs many orders of magnitude more computational effort than NS to find the evaporation transition and almost two orders of magnitude more computational effort to find the melting transition. (A similar increase in computational efficiency compared with parallel tempering was found for LJ clusters [10] and hard spheres [11, 22].)

Finally, in Figure ?? we show the phase diagram for 64 particles of Lennard Jonesium as calculated using NS with $K=640, L=1.6 \times 10^{5}$. Comparison with the literature phase diagrams for $\sim 500$ particles confirms excellent agreement with the literature values for the evaporation transition [24] and also the solid-liquid and high pressure solid-vapour transitions [23]. Below the triple point, we observe slower convergence with respect to $L$ towards literature values of the sublimation transition [25]. We also find the beginning of the Widom-line: the shallow line of heat capacity maxima that extends into the supercritical region. The Widom-line and our method for estimating the critical point are described in the SM [? ]. 


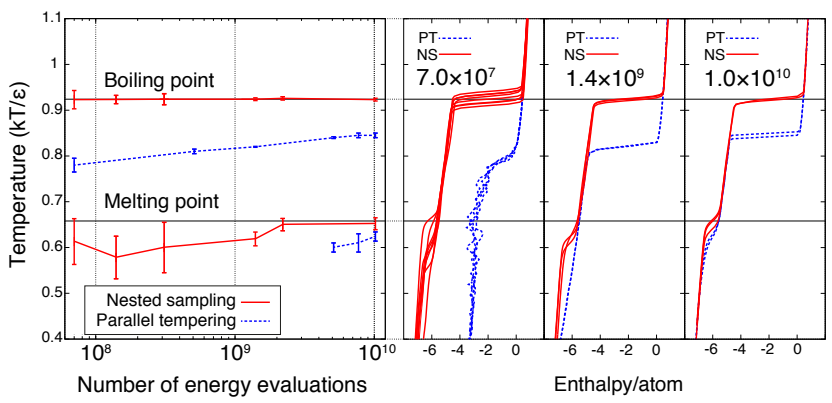

FIG. 2. Performance comparison of NS and PT. Sixty-four Lennard-Jones particles were simulated at a pressure of 0.027 (Lennard-Jones units). Both NS and PT simulations were initialised from the vapour phase. PT was performed using 128 equispaced temperature values in the range $[0.4,1.4]$. The left panel shows the estimated transition temperatures as a function of computational cost while the right panel shows the mean enthalpy as a function of temperature corresponding to three selected values of the cost.

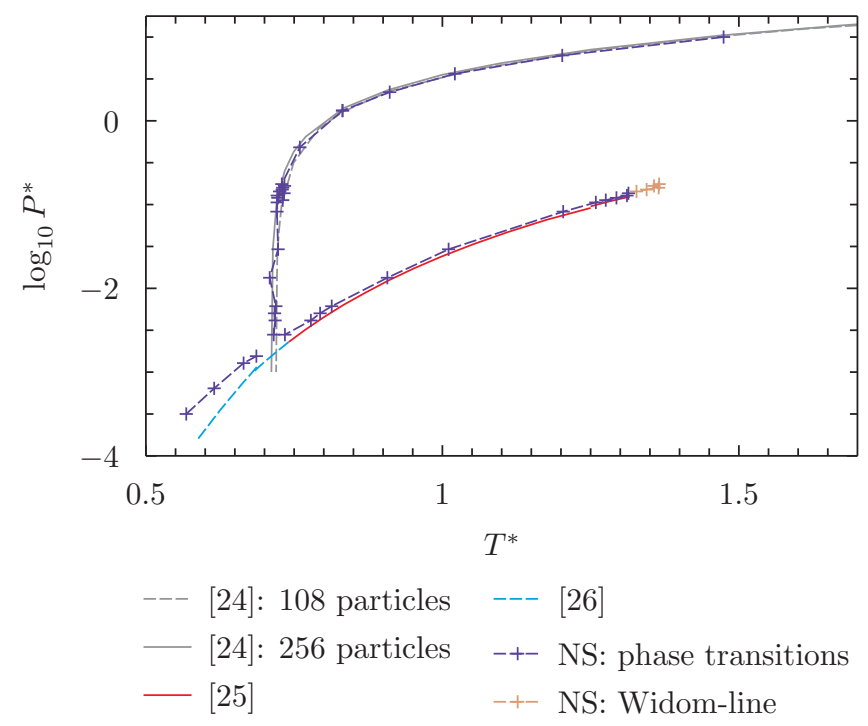

FIG. 3. Phase diagram for $N=64$ Lennard-Jones particles as calculated using NS, with comparison to the literature $(N \approx$ 500) phase diagram, as described in the text.

\section{RESULTS}

\section{A. Aluminium}

In this section we apply the new algorithm to several empirical models of aluminium in order to demonstrate the capability of nested sampling to find solid-solid phase transitions without any prior knowledge of the crystal structures or even the existence of multiple stable phases. Furthermore, although the particular off-the-shelf models we use here do not reproduce the experimentally determined phase diagram of the material everywhere, the fact that nested sampling allows a direct calculation of the entire phase diagram means that in the future one could automate the optimisation of potentials to match the experimental phase diagram.

As one of the most commonly used metals, the thermodynamic properties of aluminium have been extensively studied. The melting line of aluminium has been measured up to $125 \mathrm{GPa}$ [26-29], with good agreement between the different experimental techniques. Theoretical calculations have also been performed using embeddedatom type potentials [35-42] and ab initio methods [4345], the latter providing melting temperatures up to 350 GPa [46]. At ambient conditions aluminium crystallises in the face-centred-cubic (fcc) structure, but a phase transition to the hexagonal-close-packed (hcp) structure at $217 \mathrm{GPa}$ has been revealed by X-ray diffraction experiments [47] and the body-centred-cubic (bcc) phase has been also produced in laser-induced microexplosions [48]. The critical points of most metals are not amenable to conventional experimental study and thus estimation of their properties is usually based upon empirical relationships between the critical temperature and other measured thermodynamic properties. In the case of aluminium these result in predictions in a wide temperature and pressure range [30-33].

We chose four widely used models all based on the embedded-atom method (EAM): (1) the model developed by Liu et al. [41] (LEA-EAM), which is an improved version of the original potential of Ercolessi and Adams [40], (2) the model developed by Mishin et al. [42] using both experimental and $a b$ initio data (MishinEAM), (3) the EAM of Mei and Davenport [38] (MDEAM) and (4) the recently modified version of the MDEAM, reparametrised by Jasper et al. to accurately reproduce the DFT energies for $\mathrm{Al}$ clusters and nanoparticles of various sizes (NPB-EAM) [49].

The phase diagrams for all four models based on NS simulations with 64 particles are shown in Figure 2. The resulting critical parameters vary over a wide range for the different models. Above the critical point we observe the Widom-line, indicated by those points not linked by a solid line. Heat capacity maxima corresponding to the Widom-line become broader away from the critical point, as indicated by the larger error bars. The Widom-line and our method for estimating the critical point are described in the SM [?].

The melting lines are in a good agreement with the available experimental data up to the pressure value $p \approx 25 \mathrm{GPa}$. Above that pressure, the melting curves diverge from the experimental results, except for the MDEAM potential, which reproduces the melting curve remarkably well.

At higher pressures small peaks appear on the heat capacity curves below the melting temperature for all models indicating solid-solid phase transitions (see appendix C). We post-processed the samples from the NS simulations. As expected, the fcc structure was found to be stable at low pressures in all four models. However, the models differ markedly in their predictions at high pressures. The only commonality between the predicted high pressure solid phase diagrams is that the maximum 


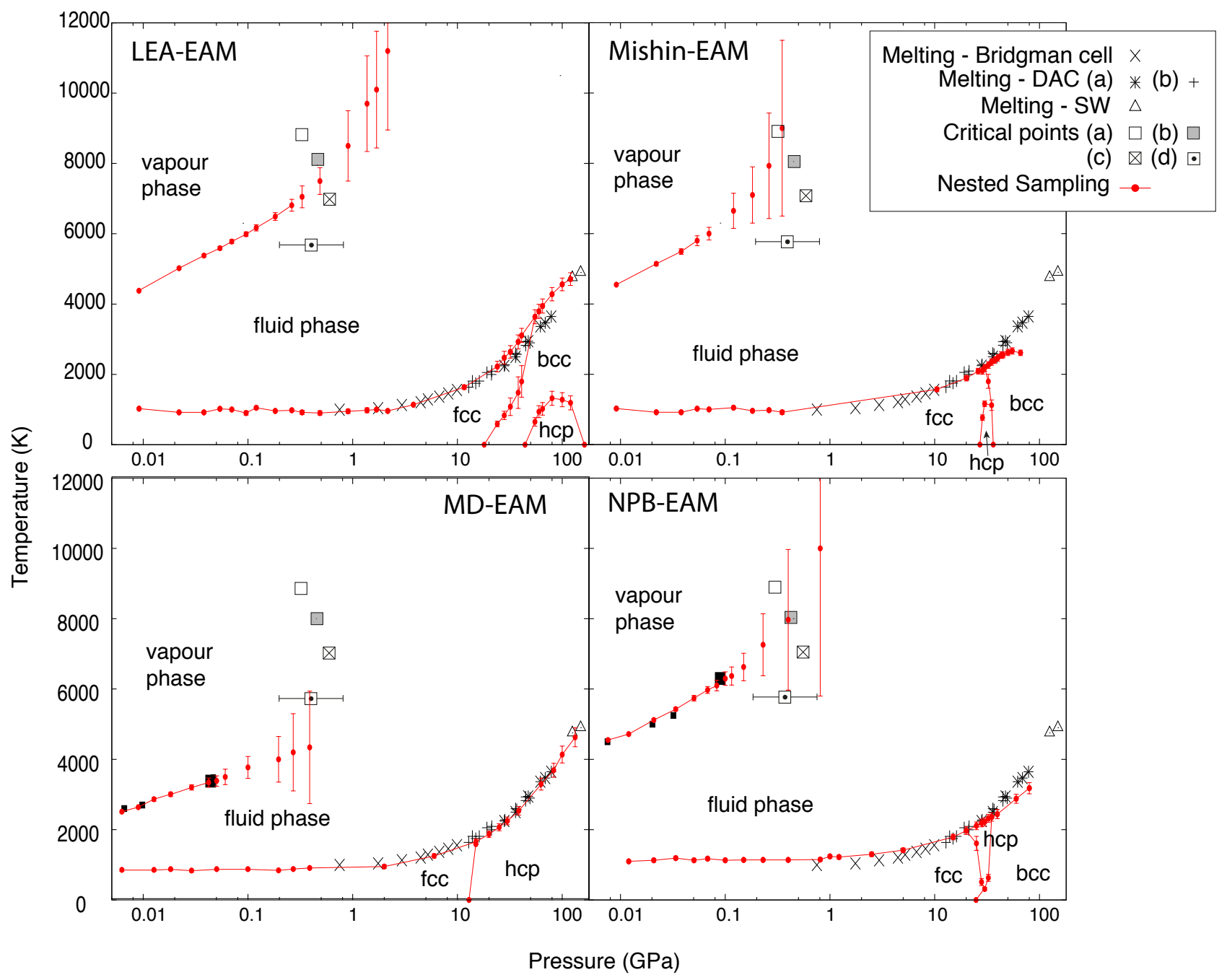

FIG. 4. Phase diagrams corresponding to four EAM models of aluminium. Red symbols show the NS results, the error bars are calculated as the width at half maximum of the peaks on the heat capacity curves. On the boiling line points are connected by a solid line up to the critical point. (The method we used to estimate the critical point is described in the SM [? ].) Black symbols show experimental melting points measured with Bridgman cells [26], with Diamond anvil cells (DAC (a) [27] and (b) [28]) and shock waves (SW) [29]. Different square symbols show estimates of the critical point from experiments, (a) [30], (b) [31], (c) [32] and (d) [33]. For NPB-EAM and MD-EAM large black squares show the critical point and smaller black squares show the evaporation temperatures, all calculated using Gibbs ensemble Monte Carlo [34]. At pressures below the critical point, NS parameters $K=800$ and $L=3000$ were used (the total number of energy evaluations was $3 \times 10^{9}$ for each pressure), while runs at pressures where solid-solid transitions are present required $K=3200$ and $L=15000$ (total number of energy evaluations were $4 \times 10^{10}$ ).

predicted stable pressure for the fcc structure is far too low, both in comparison with experiment and density functional theory $[47,50,51]$.

\section{B. NiTi}

Finally, in order to demonstrate that NS is applicable to more complex problems, we show results for a material of current scientific interest, the NiTi shape memory alloy $[52,53]$. The shape memory effect relies on the structural phase transition from the high temper- ature austenitic phase to the low temperature martensitic phase [54]. Studying this transition is particularly challenging with traditional free energy methods because the austenitic phase does not correspond to a local minimum of the potential energy surface. Figure 3 shows the pressure-temperature-composition phase diagram corresponding to a recent EAM model $[55,56]$ as computed with NS. The phase transition temperatures are within $50 \mathrm{~K}$ of the experimental values and reproduce the trend with compositional change. We predict a decreasing transition temperature with increasing pressure. It is notable that this potential successfully reproduces the marten- 


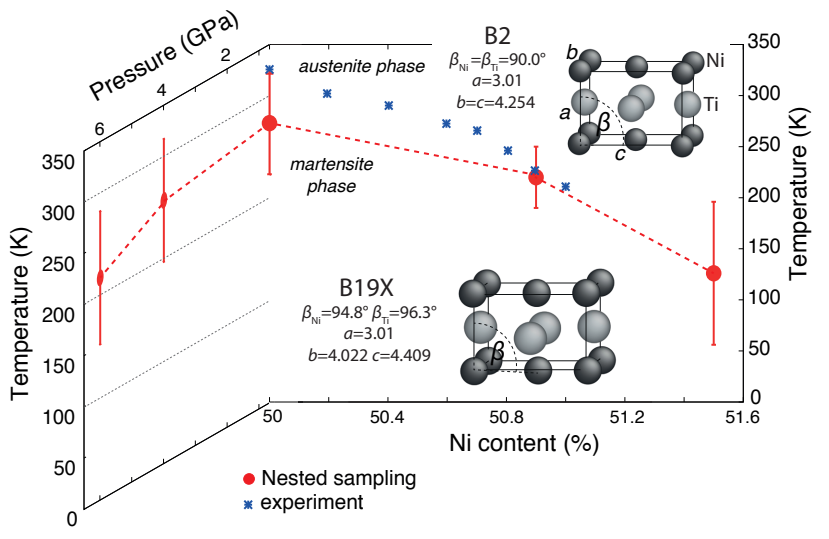

FIG. 5. NiTi martensitic phase transition as a function of $\mathrm{Ni}$ content (at $0.66 \mathrm{GPa}$ ) and pressure (at 1:1 composition). The simulation cell contained 64 atoms in the cases of the $50 \%$ and $51.6 \% \mathrm{Ni}$ compositions and 108 atoms in the case of $50.8 \% \mathrm{Ni}$ content. NS parameters were $K=1920, L=10^{5}$, each data point used $10^{10}$ energy evaluations, and $\mathrm{Ni}-\mathrm{Ti}$ swap moves were also included in the MC. Experimental results are taken from [57].

sitic transition temperature, despite the fact that the minimum enthalpy structure for the potential is different to the structure observed both experimentally and in DFT: here the lowest enthalpy structure (which we label B19X) is orthorhombic (see the SM [? ] for a description of the low enthalpy structures we identified). Thus it appears that the austenite-martensite transition temperature is not sensitive to the detailed geometry and ordering of the lowest enthalpy structures. Such empirical potentials can therefore be useful tools for studying this transition in the future.

\section{CONCLUSION AND OUTLOOK}

In summary, we have extended the nested sampling algorithm to allow simulations using fully flexible periodic boundary conditions at fixed pressure and demonstrated how it can be used to determine pressure-temperaturecomposition phase diagrams. In contrast to existing methods for comparing specific phases, NS explores the entire configuration space without requiring any prior knowledge about the structures of different solid phases with the only necessary input being the composition and the desired pressure and temperature ranges. This makes it the method of choice for exploring the pressuretemperature-composition space, which is the next unexplored realm naturally following much recent work in crystal structure exploration at zero temperature. Since the algorithm is run independently for different pressures and compositions, and also has excellent parallel scaling up to a number of processors equal to the number of simultaneous samples, it might even be possible to run it on ab initio models on exascale computers. Furthermore, we suggest NS is eminently suitable for validating materials models, and in the future could even play a role in the automatic optimisation of empirical models.

\section{ACKNOWLEDGMENTS}

RJNB acknowledges support from the EPSRC. LBP acknowledges support from St. Catharine's College, Cambridge and to the Royal Society. APB acknowledges support from Magdalene College, Cambridge, the Leverhulme Trust and the Isaac Newton Trust. GC acknowledges EPSRC grant EP/J010847/1. Computer time was provided via the UKCP consortium funded by EPSRC grant ref. EP/K013564/1 and via the Darwin supercomputer in the University of Cambridge High Performance Computing Service funded under EPSRC grant EP/J017639/1. Data from this publication can be found at https: //www.repository.cam.ac.uk/handle/ $1810 / 255091$.

\section{Appendix A: Ideal gas contribution to the partition function}

In this appendix we show that the ideal gas contribution to the partition function (8) asymptotically approaches zero for any positive minimum cell depth $d_{0}$, in the limit $k_{\mathrm{B}} T / p V_{0} \rightarrow 0$.

The ideal gas contribution to the partition function (8) is

$$
\int_{D\left(\mathbf{h}_{0}\right)>d_{0}} d \mathbf{h}_{0} \delta\left(\operatorname{det} \mathbf{h}_{0}-1\right) \int_{V_{0}}^{\infty} d V V^{N} \int_{(0,1)^{3 N}} d \mathbf{s} e^{-\beta p V}
$$

We begin by noting that the exponential term does not depend on $E\left(\mathbf{s}, \mathbf{h}_{0}, V\right)$, and therefore $\int_{(0,1)^{3 N}} d \mathbf{s}=1$. Thus we have

$$
\begin{array}{r}
\int_{D\left(\mathbf{h}_{0}\right)>d_{0}} d \mathbf{h}_{0} \delta\left(\operatorname{det} \mathbf{h}_{0}-1\right) \int_{V_{0}}^{\infty} d V V^{N} \int_{(0,1)^{3 N}} \mathrm{~d} \mathbf{s} e^{-\beta p V} \\
=\int_{D\left(\mathbf{h}_{0}\right)>d_{0}} d \mathbf{h}_{0} \delta\left(\operatorname{det} \mathbf{h}_{0}-1\right) \int_{V_{0}}^{\infty} d V V^{N} e^{-\beta p V}
\end{array}
$$

The integral over volume $V$ evaluates to

$$
\int_{V_{0}}^{\infty} d V V^{N} e^{-\beta p V}=\frac{1}{(\beta p)^{N+1}} \Gamma\left(N+1, \beta p V_{0}\right)
$$

where $\Gamma\left(N+1, \beta p V_{0}\right)$ is the upper incomplete gamma function.

Finally we define the function $A\left(d_{0}\right)$ to be equal to the integral over $\mathbf{h}_{0}$

$$
A\left(d_{0}\right)=\int_{D\left(\mathbf{h}_{0}\right)>d_{0}} d \mathbf{h}_{0} \delta\left(\operatorname{det} \mathbf{h}_{0}-1\right) .
$$

The function $A\left(d_{0}\right)$ is finite for any positive value of $d_{0}$, $A(1)=0$ and $A\left(d_{0}\right)$ diverges in the limit $d_{0} \rightarrow 0$. In 
the orthorhombic case, where all angles of the simulation cell are equal to $\frac{\pi}{2}, A\left(d_{0}\right)=\frac{9}{2}\left(\log d_{0}\right)^{2}$, with $A=1$ at $d_{0} \approx 0.62$. However at any positive value of $d_{0}$ the contribution to the partition function (8) due to volumes greater than $V_{0}$ goes to zero in the limit $k_{\mathrm{B}} T / p V_{0} \rightarrow 0$ because $\Gamma\left(N+1, \beta p V_{0}\right) \rightarrow 0$ in the same limit.

\section{Appendix B: Identifying solid-solid phase transitions}

The locations of phase transitions are determined solely by looking at the peaks in the heat capacity. Next, we inspect the system at temperatures either side of the phase transition. Specific phases can be identified in the following way. If no appropriate order parameter is to hand, then one picks a number of random configurations from the output of nested sampling, chosen according to their thermal weights $\Delta \chi_{i} e^{-\beta H_{i}}$, and inspects them by eye. If an appropriate order parameter is known, one can compute the free energy landscape for that order parameter. Here one proceeds by binning the weights $\Delta \chi_{i} e^{-\beta H_{i}}$ of all configurations, according to the order parameter, to create a partial sum $\Delta_{j}=\sum \Delta \chi_{i} e^{-\beta H_{i}}$ for each bin $j$. The free energy for each bin can then be computed as $F_{j}=-\frac{1}{\beta}\left[\log \left(\Delta_{j}\right)+\log \left(\frac{\beta p}{N !}\right)+\frac{3 N}{2} \log \left(\frac{2 \pi m}{\beta h^{2}}\right)\right]$. In fact, simply calculating the expected enthalpy at the phase transition, and then examining the order parameter values for output configurations around that enthalpy is often sufficient to identify the crystal structures.

An example of the latter approach is shown in Figure 4 for the Mishin-EAM potential, which compares the enthalpies and $Q_{6}$ bond order parameter values for nested sampling output configurations at three different pressures. At $p=25.0 \mathrm{GPa}$ no phase transition occurs, and only fcc configurations are present. At $p=34.9 \mathrm{GPa}$ a first order phase transition occurs at the average enthalpy marked by the vertical dashed line. At that enthalpy there is a clear transition between two basins, from a first basin that corresponds to the bcc structure, to a second that corresponds to the hcp structure. Finally, at $p=37.5 \mathrm{GPa}$ no phase transition occurs and so there is no peak in the heat capacity. At this pressure the bcc structure is stable at all temperatures below the melting point. Nevertheless, the hcp structure is clearly visible as a metastable structure.
[1] A. Z. Panagiotopoulos, Mol. Phys. 61, 813 (1987).

[2] D. A. Kofke, Mol. Phys 78, 1331 (1993).

[3] J. Skilling, AIP Conf. Proc. 735, 395 (2004).

[4] J. Skilling, Bayesian Anal. 1, 833 (2006).

[5] R. H. Swendsen and J. S. Wang, Phys. Rev. Lett. 57, 2607 (1986).

[6] D. D. Frantz, D. L. Freemann, and J. D. Doll, J. Chem. Phys. 93, 2769 (1990).

[7] F. Wang and D. P. Landau, Phys. Rev. Lett. 86, 2050 (2001).

[8] M. Allen and D. Tildesley, Computer Simulation in Chemical Physics, Nato Science Series C: (Springer Netherlands, 2012).

[9] F. Feroz and M. P. Hobson, Mon. Not. R. Astron. Soc. 384, 449 (2008).

[10] L. B. Pártay, A. P. Bartók, and G. Csányi, J. Phys. Chem. B 114, 10502 (2010).

[11] L. B. Pártay, A. P. Bartók, and G. Csányi, Phys. Rev. E 89, 022302 (2014).

[12] N. S. Burkoff, C. Várnai, S. A. Wells, and D. L. Wild, Biophys. J. 102, 878 (2012).

[13] H. Do, J. D. Hirst, and R. J. Wheatley, J. Chem. Phys. 135, 174105 (2011).

[14] H. Do, J. D. Hirst, and R. J. Wheatley, J. Phys. Chem. 116, 4535 (2012).

[15] H. Do and R. J. Wheatley, J. Chem. Theory Comput. 9, 165 (2013).

[16] S. Martiniani, J. D. Stevenson, D. J. Wales, and D. Frenkel, Phys. Rev. X 4, 031034 (2014).

[17] B. J. Brewer, L. B. Pártay, and G. Csányi, Stat. Comput. 21, 649 (2010).

[18] G. J. Martyna, D. J. Tobias, and M. L. Klein, J. Chem. Phys. 101, 4177 (1994).

[19] S. Brooks, A. Gelman, G. Jones, and X.-L. Meng, Hand- book of Markov Chain Monte Carlo (CRC press, 2011).

[20] D. Frenkel and B. Smit, Understanding Molecular Simulation: From Algorithms to Applications (Elsevier Science, 2001).

[21] M. Tuckerman, Statistical Mechanics and Molecular Simulations (Oxford University Press, 2008).

[22] G. Odriozola, J. Chem. Phys. 131, 144107 (2009).

[23] G. C. McNeil-Watson and N. B. Wilding, J. Chem. Phys. 124, 064504 (2006).

[24] D. A. Kofke, J. Chem. Phys. 98, 4149 (1993).

[25] R. Agrawal and D. A. Kofke, Mol. Phys. 85, 43 (1995).

[26] D. Errandonea, J. App. Phys. 108, 033517 (2010).

[27] R. Boehler and M. Ross, Earth Planet. Sci. Lett. 153, 1 (1997).

[28] A. Hänström and P. Lazor, J. Alloys Compd. 305, 209 (2000).

[29] J. W. Shaner, J. M. Brown, and R. G. McQueen, "Melting of metals above 100 gpa," in High Pressure in Science and Technology, edited by C. Homan, R. K. MacCrone, and E. Whalley (Amsterdam: North-Holland, 1984) pp. 137-141.

[30] A. A. Likalter, Physica A 311, 137 (2002).

[31] V. Fortov and I. Iakubov, The Physics of Non-ideal Plasma (World Scientific, 2000).

[32] D. A. Young and A. B. J, Phys. Rev. A 3, 364 (1971).

[33] P. Renaudin, C. Blancard, J. Clérouin, G. Faussurier, P. Noiret, and V. Recoules, Phys. Rev. Lett. 91, 075002 (2003).

[34] D. Bhatt, A. W. Jasper, N. E. Schultz, J. I. Siepmann, and D. G. Truhlar, J. Am. Chem. Soc. 128, 4224 (2006).

[35] S. M. Foiles, M. I. Baskes, and M. S. Daw, Phys. Rev. B 33, 7983 (1986).

[36] A. F. Voter and S. P. Chen, Mater. Res. Soc. Symp. Proc. 82, 175 (1987). 


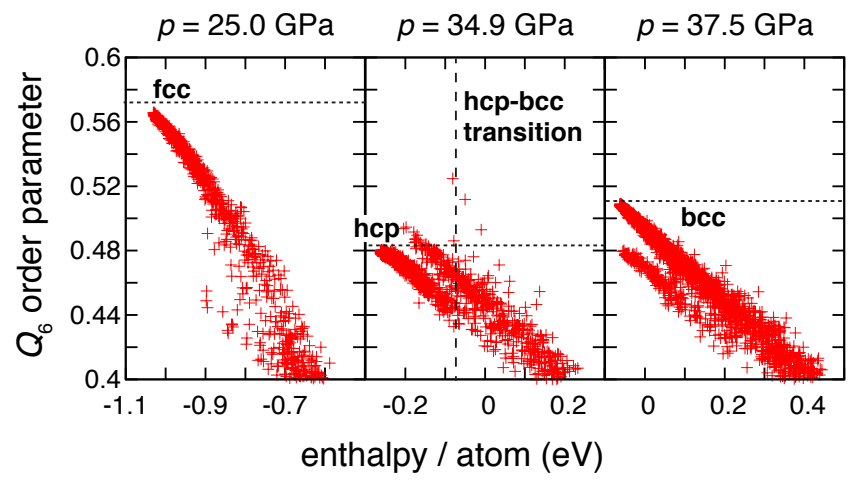

FIG. 6. Identification of solid phases from inspection of an order parameter: Mishin-EAM potential for aluminium. These plots compare the enthalpies and $Q_{6}$ bond order parameter values for nested sampling output configurations at three different pressures. Nested sampling proceeds towards lower enthalpies, thus from right to left in each plot. Horizontal dotted lines show the bond order parameters for the perfect fcc, bcc and hcp phases, and the vertical dashed line shows the expected enthalpy at the solid-solid phase transition, which was located by inspecting the heat capacity and observing a peak. These results are discussed in the text.

[37] D. J. Oh and R. A. Johnson, J. Mater. Res. 3, 471 (1988). [38] J. Mei and J. W. Davenport, Phys. Rev. B 46, 21 (1992).

[39] J. R. Morris, C. Z. Wang, K. M. Ho, and C. T. Chan, Phys. Rev. B 49, 3109 (1994).

[40] F. Ercolessi and J. B. Adams, Europhys. Lett. 26, 583 (1994).
[41] X.-Y. Liu, F. Ercolessi, and J. B. Adams, Model. Simul. Mater. Sci. Eng. 12, 665 (2004).

[42] Y. Mishin, D. Farkas, M. J. Mehl, and D. A. Papaconstantopoulos, Phys. Rev. B 59, 3393 (1999).

[43] G. A. de Wijs, G. Kresse, and M. J. Gillan, Phys. Rev. B 57, 8223 (1998).

[44] L. Vočadlo and D. Alfè, Phys. Rev. B 65, 214105 (2002).

[45] D. Alfè, L. Vočadlo, G. D. Price, and M. J. Gillan, J. Phys. Condens. Matter 16, S973 (2004).

[46] J. Bouchet, F. Bottin, G. Jomard, and G. Zérah, Phys. Rev. B 80, 094102 (2009).

[47] Y. Akahama, M. Nishimura, K. Kinoshita, H. Kawamura, and Y. Ohishi, Phys. Rev. Lett. 96, 045505 (2006).

[48] A. Vailionis, E. G. Gamaly, V. Mizeikis, W. Yang, A. V. Rode, and S. Juodkazis, Nature Commun. 2, 445 (2011).

[49] A. W. Jasper, N. E. Schultz, and D. G. Truhlar, J. Phys. Chem. B 109, 3915 (2005).

[50] J. C. Boettger and S. B. Trickey, Phys. Rev. B 53, 3007 (1996).

[51] G. Sin'ko and N. Smirnov, J. Phys. Condens. Matter 14, 6989 (2002).

[52] Y. Ji, D. Wang, X. Ding, K. Otsuka, and X. Ren, Phys. Rev. Lett. 114, 055701 (2015).

[53] W. J. Buehler, J. W. Gilfrich, and R. C. Wiley, J. Appl. Phys. 34, 1475 (1963).

[54] K. Bhattacharya, Microstructure of martensite: why it forms and how it gives rise to the shape-memory effect (Oxford University Press, 2003).

[55] Y. Zhong, K. Gall, and T. Zhu, J. App. Phys. 110, 033532 (2011).

[56] R. Mirzaeifar, K. Gall, T. Zhu, A. Yavari, and R. DesRoches, J. Appl. Phys. 115, 194307 (2014).

[57] J. Frenzel, E. George, A. Dlouhy, C. Somsen, M.-X. Wagner, and G. Eggeler, Acta Mater. 58, 3444 (2010).

[58] R. J. N. Baldock, Classical Statistical Mechanics with Nested Sampling, Ph.D. thesis, University of Cambridge (2014). 